\title{
Synthesis of new pyrimidine derivatives and their antiproliferative activity against selected human cancer cell lines
}

\author{
S. M. Awad • O. A. Fathalla · J. Wietrzyk • \\ M. Milczarek • A. M. Soliman • Mosaad S. Mohamed
}

Received: 14 February 2013/Accepted: 11 June 2013/Published online: 27 June 2013

(C) The Author(s) 2013. This article is published with open access at Springerlink.com

\begin{abstract}
Amino-2-thiouracil (1) was condensed with benzenesulfonyl chloride and $p$-toluenesulfonyl chloride in presence of pyridine as an acid binder to give sulfonamides $\mathbf{2 a}, \mathbf{b}$, which could be methylated in basic medium to give methylmercapto derivatives $\mathbf{3 a}, \mathbf{b}$, which in turn reacted with bromine in glacial acetic acid to yield 5-bromo derivatives $\mathbf{4 a}, \mathbf{b}$. On the other hand, compounds $\mathbf{2 a}$, b were cyclocondensed with monochloroacetyl chloride, $p$-tolualdehyde in glacial acetic acid/ pyridine, and ethyl bromoacetate to give the corresponding thiazolopyrimidines $\mathbf{5 a}$, $\mathbf{b}, \mathbf{6 a}, \mathbf{b}$, and $\mathbf{7 a}, \mathbf{b}$, respectively; also compounds $\mathbf{2 a}, \mathbf{b}$ were hydrazinolyzed to compounds $\mathbf{8 a}, \mathbf{b}$, which could be cyclized to triazolopyrimidines $\mathbf{9 a}, \mathbf{b}$ in presence of formic acid. They could also be condensed with $p$-anisaldehyde to give hydrazones 10a, b. In another pathway, compounds $\mathbf{2 a}$, b were reacted with monochloroacetic acid in basic medium to give acetic acid derivatives $11 \mathbf{a}, \mathbf{b}$. It can be deduced from the preliminary screening results that the cell lines most sensitive to the antiproliferative activity of tested compounds are human liver HEPG2 and colon cancer HT-29. All selected compounds exhibited moderate to strong growth inhibition activity against the HEPG2 cell line with $50 \%$ inhibitory concentration $\left(\mathrm{IC}_{50}\right)$ ranging between 1 and $10 \mu \mathrm{g} / \mathrm{ml}$. The most active compounds, which revealed antiproliferative activity also against human colon HT-29 and breast MCF7 cell lines, were $\mathbf{3 a}, \mathbf{3 b}, \mathbf{4 a}$, and $\mathbf{1 0 a}$.
\end{abstract}

S. M. Awad · M. S. Mohamed

Pharmaceutical Organic Chemistry Department, Faculty of Pharmacy, Helwan University, Helwan, Egypt

O. A. Fathalla $(\bowtie) \cdot$ A. M. Soliman

Therapeutic Chemistry Department, National Research Centre, Dokky, P.O. Box 12622, Cairo, Egypt

e-mail: omarfathalla@yahoo.com

J. Wietrzyk · M. Milczarek

Department of Experimental Oncology, Institute of Immunology and Experimental Therapy, 12, Rudolf Weigl St., 53-114 Wroclaw, Poland 
Keywords New pyrimidine derivatives - 6-Sulfonamide derivatives - Thiazole Triazole $\cdot$ Antiproliferative activity

\section{Introduction}

5-Fluorouracil (5-FU) is one of the oldest chemotherapy drugs [1] and has been in use for decades. It is an active medicine against many cancers including colon, rectal, pancreas, head, neck, and liver cancers [2]. It is also used topically as a cream or solution to treat basal cell cancer of skin and actinic keratoses, thus becoming the first prototype of pyrimidines in this field. Analogously, some 5-halogenated thiouracils have been synthesized and screened for anticancer activity. It has been reported that the tested compounds had comparable activity to that of uracils [3]. From 1957 to 2011, different research laboratories investigated the anticancer activity of some 5- and 6-substituted 2-thiouracils and reported that the tested compounds were found to inhibit DNA synthesis [4-11]. Based on these findings, the present work aims to synthesize a new group of pyrimidine compounds incorporated with different heterocycles to investigate whether the resulting compounds have better biological activity as antiproliferative agents against cancer, since all the selected pyrimidine derivatives were soluble in dimethyl sulfoxide (DMSO) at sufficient concentrations to allow cell experiments. The in vitro biological activity of these compounds was evaluated by their growth inhibitory potency against different cancer cell lines. The cytotoxic potency of compounds 3a, $3 \mathbf{b}, 4 \mathbf{a}, 5 \mathbf{b}, \mathbf{6 a}, 7 \mathbf{b}, \mathbf{8 a}, 9 \mathbf{b}, \mathbf{1 0 a}$, and 11a was studied in comparison with the known anticancer drugs 5-FU and cisplatin (CIS).

In continuation of our work on the synthesis of some 2-thiouracils [12], we report here the synthesis of novel thiouracil derivatives based on the diverse medicinal uses and biological activities of thiouracils as anticancer [12], antithyroid [13], and anti-infective agents [13-15]. In this work we screened some of the prepared compounds as anticancer agents in analogy to 5-FU, and we also develop a program to incorporate the thiouracil nucleus into thiazole and triazole nuclei to compare their activities with 5-FU as a reference standard, finally concluding that 5- and 6-substituted 2-thiouracils may have activity comparable to 5-FU.

\section{Chemistry}

All melting points are uncorrected and were determined in capillary tube on a Boetius melting-point microscope. Infrared (IR) spectra $\left(\mathrm{KBr}, \mathrm{cm}^{-1}\right)$ were recorded from $\mathrm{KBr}$ discs. ${ }^{1} \mathrm{H}$ nuclear magnetic resonance (NMR) spectra were obtained on a Joel EX 270-MHz spectrometer with tetramethylsilane (TMS) as internal standard. Mass spectra were recorded on a SSQ 7000 mass spectrometer at $70 \mathrm{eV}$. All reactions were followed and checked by thin-layer chromatography (TLC) using chloroform/methanol (3:1), and spots were examined by ultraviolet (UV) lamp. 


\section{6-Amino-2-thiouracil (1)}

This compound was prepared according to the reported method [16, 17].

$N$-(6-Oxo-2-thioxo-1,2,3,6-tetrahydropyrimidin-4-yl)benzenesulfonamide and 4-methyl- $N$-(6-oxo-2-thioxo-1,2,3,6-tetrahydropyrimidin-4yl)benzenesulfonamide (2a, b)

A mixture of 1 (1.42 $\mathrm{g}$ of $0.05 \mathrm{~mol})$, benzenesulfonyl chloride $(0.88 \mathrm{~g}$ of $0.05 \mathrm{~mol})$ or $p$-toluenesulfonyl chloride $(0.95 \mathrm{~g}$ of $0.05 \mathrm{~mol})$, and pyridine $(0.4 \mathrm{ml})$ in methanol $(25 \mathrm{ml})$ was refluxed for $12 \mathrm{~h}$, then cooled; the reaction mixture was poured into ice $/ \mathrm{HCl}$, and the resulting solid formed was filtered off, washed with water, dried, and recrystallized from dimethylformamide (DMF)/water.

$\mathrm{N}$-[2-(Methylsulfanyl)-6-oxo-1,6-dihydropyrimidin-4-yl]benzenesulfonamide and 4-methyl- $N$-[2-(methylsulfanyl)-6-oxo-1,6-dihydropyrimidin-4yl]benzenesulfonamide $(\mathbf{3 a}, \mathbf{b})$

An amount $(0.05 \mathrm{~mol})$ of $\mathbf{2 a}, \mathbf{b}$ was dissolved in $25 \mathrm{ml} 10 \%$ alc. $\mathrm{NaOH}$ with stirring, then methyl iodide $(0.05 \mathrm{~mol})$ was added with stirring for $4 \mathrm{~h}$, then the reaction mixture was neutralized with dil. $\mathrm{HCl}$, filtered, dried, and recrystallized from methanol.

$\mathrm{N}$-[5-Bromo-2-(methylsulfanyl)-6-oxo-1,6-dihydropyrimidin-4yl]benzenesulfonamide and $N$-[5-bromo-2-(methylsulfanyl)-6-oxo-1,6dihydropyrimidin-4-yl)-4-methylbenzenesulfonamide (4a, b)

An amount $(0.05 \mathrm{~mol})$ of $\mathbf{3 a}, \mathbf{b}$ was stirred with $0.05 \mathrm{~mol}$ bromine in $25 \mathrm{ml}$ glacial acetic acid for $24 \mathrm{~h}$, then the produced solid was filtered off, washed several times with water, dried, and recrystallized from methanol.

$N$-(2,5-Dioxo-2,3,8,8a-tetrahydro-5- $H$-[1,3]thiazolo[3,2- $a$ ]pyrimidin-7yl)benzenesulfonamide and $N$-(2,5-dioxo-2,3,8,8a-tetrahydro-5- $H$ $[1,3]$ thiazolo[3,2-a]pyrimidin-7-yl)-4-methylbenzenesulfonamide (5a, b)

To an ice-cold solution of $\mathbf{2 a}, \mathbf{b}(0.01 \mathrm{~mol})$ in $40 \mathrm{ml}$ dry DMF, triethylamine $(0.01 \mathrm{~mol})$ and chloroacetyl chloride $(0.01 \mathrm{~mol})$ were added successively. The reaction mixture was heated in a water bath at $70{ }^{\circ} \mathrm{C}$ for $6 \mathrm{~h}$, cooled, poured into ice/cold water, and extracted with $3 \times 30 \mathrm{ml}$ methylene chloride; the organic layer was dried on anhydrous sodium sulfate and filtered off, and the filtrate was evaporated to dryness. The residue was washed with $10 \%$ sodium carbonate solution, and the undissolved solid was filtered off, and crystallized from DMF/ water. 
$\mathrm{N}$-[(2E)-2-(4-Methylbenzylidene)-3,5-dioxo-2,3,8,8a-tetrahydro-5H-

$[1,3]$ thiazolo[3,2-a]pyrimidin-7-yl]benzenesulfonamide and 4-methyl- $N$-[(2E)-

2-(4-methylbenzylidene)-3,5-dioxo-2,3,8,8a-tetrahydro-5H-[1,3]thiazolo[3,2-

a]pyrimidin-7-yl]benzenesulfonamide $(\mathbf{6 a}, \mathbf{b})$

A mixture of $\mathbf{2 a}, \mathbf{b}(0.001 \mathrm{~mol})$ and $p$-tolualdehyde $(0.001 \mathrm{~mol})$ in $30 \mathrm{ml}$ glacial acetic acid/acetic anhydride mixture was refluxed for $7 \mathrm{~h}$, then cooled, and the produced solid was filtered off, dried, and crystallized from DMF/water.

$N$-(3,5-Dioxo-2,3,8,8a-tetrahydro-5 $H$-[1,3]thiazolo[3,2-a]pyrimidin-7-

yl)benzenesulfonamide and $\mathrm{N}$-(3,5-dioxo-2,3,8,8a-tetrahydro-5H-

[1,3]thiazolo[3,2-a]pyrimidin-7-yl)-4-methylbenzenesulfonamide (7a, b)

To an ice-cold solution of $\mathbf{2 a}, \mathbf{b}(0.01 \mathrm{~mol})$ in $40 \mathrm{ml}$ dry DMF, triethylamine $(0.01 \mathrm{~mol})$ and ethyl bromoacetate $(0.01 \mathrm{~mol})$ were added successively. The reaction mixture was heated in a water bath at $70{ }^{\circ} \mathrm{C}$ for $6 \mathrm{~h}$, cooled, poured into ice/cold water, and extracted with methylene chloride $(3 \times 30 \mathrm{ml})$; the organic layer was dried on anhydrous sodium sulfate and filtered off, and the filtrate was evaporated to dryness. The residue was washed with $10 \%$ sodium carbonate solution, and the undissolved solid was filtered off, and crystallized from DMF/ water.

\section{$2 a$}

Yield $75 \%$, m.p. $274{ }^{\circ} \mathrm{C}$, Anal. Calcd. $\mathrm{C}_{10} \mathrm{H}_{9} \mathrm{~N}_{3} \mathrm{O}_{3} \mathrm{~S}_{2}$ (283.33): C, 42.34; H, 3.20; N, 14.83. Found: C, 42.09; H, 3.45; N, 14.78; IR (KBr, $\left.\mathrm{cm}^{-1}\right): 3,300(\mathrm{NH}), 3,100$ (CH aromatic), 1,668 (C=O of thiouracil), 1,140, 1,320 $\left(\mathrm{SO}_{2}\right), 1,270(\mathrm{C}=\mathrm{S}) ;{ }^{1} \mathrm{H}$ NMR (DMSO- $\left.d_{6}\right) 6.8(1 \mathrm{H}, \mathrm{s}$, thiouracil), 7.1-7.4 $(5 \mathrm{H}, \mathrm{m}), 9.8,10.2,10.5(3 \mathrm{NH}, \mathrm{s}$, exchangeable with $\left.\mathrm{D}_{2} \mathrm{O}\right)$; MS: $m / z 283.3\left(\mathrm{M}^{+}, 27.1 \%\right)$.

\section{$2 b$}

Yield $57 \%$, m.p. $288{ }^{\circ} \mathrm{C}$, Anal. Calcd. $\mathrm{C}_{11} \mathrm{H}_{11} \mathrm{~N}_{3} \mathrm{O}_{3} \mathrm{~S}_{2}$ (297.36): C, 44.43; H, 3.73; N, 14.13. Found: C, 44.72; H, 3.95; N, 14.06; IR $\left(\mathrm{KBr}, \mathrm{cm}^{-1}\right): 3,350(\mathrm{NH}), 3,100$ (CH aromatic), 2,950 ( $\mathrm{CH}$, aliphatic), 1,676 ( $\mathrm{C}=\mathrm{O}$ of thiouracil), 1,143, 1,327 $\left(\mathrm{SO}_{2}\right)$, 1,276 $(\mathrm{C}=\mathrm{S})$; ${ }^{1} \mathrm{H}$ NMR (DMSO-d $) 2.5\left(\mathrm{H}_{3} \mathrm{C}-\mathrm{S}\right), 6.7(1 \mathrm{H}, \mathrm{s}$, thiouracil), 7.1-7.3 $(4 \mathrm{H}$, dd), 9.6,10.410.7 (3NH, s, exchangeable with $\left.\mathrm{D}_{2} \mathrm{O}\right)$; MS: $\mathrm{m} / z 297.4\left(\mathrm{M}^{+}, 39.3 \%\right)$.

$3 a$

Yield $63 \%$, m.p. $301{ }^{\circ} \mathrm{C}$, Anal. Calcd. $\mathrm{C}_{11} \mathrm{H}_{12} \mathrm{~N}_{3} \mathrm{O}_{3} \mathrm{~S}_{2}$ (297.36): C, 44.43; H, 3.73; N, 14.13. Found: C, $44.09 \mathrm{H}, 4.38$; N, 13.99; IR (KBr, $\left.\mathrm{cm}^{-1}\right): 3,387(\mathrm{NH}), 2,978$ (CH aliphatic), 3,167 ( $\mathrm{CH}$ aromatic), 1,665 ( $\mathrm{C}=\mathrm{O}$ of thiouracil), 1,143, 1,325 $\left(\mathrm{SO}_{2}\right)$, $1,274(\mathrm{C}=\mathrm{S}) ;{ }^{1} \mathrm{H}$ NMR (DMSO- $\left.d_{6}\right), 2.3\left(\mathrm{H}_{3} \mathrm{C}-\mathrm{S}\right), 6.9(1 \mathrm{H}, \mathrm{s}$, thiouracil), 7.1-7.3 $(5 \mathrm{H}, \mathrm{m}), 9.9,10.5\left(2 \mathrm{NH}, \mathrm{s}\right.$, exchangeable with $\left.\mathrm{D}_{2} \mathrm{O}\right)$; $\mathrm{MS}: m / z 297.4\left(\mathrm{M}^{+}, 15.8 \%\right)$. 
$3 b$

Yield $78 \%$, m.p. $361{ }^{\circ} \mathrm{C}$, Anal. Calcd. $\mathrm{C}_{12} \mathrm{H}_{14} \mathrm{~N}_{3} \mathrm{O}_{3} \mathrm{~S}_{2}$ (312.38): C, 46.13; H, 4.52; N, 13.45, Found: C, 46.44; H, 4.66; N, 13.19; IR (KBr, $\left.\mathrm{cm}^{-1}\right): 3,348(\mathrm{NH}), 2,990$ ( $\mathrm{CH}$ aliphatic), 3,177 ( $\mathrm{CH}$ aromatic), 1,685 ( $\mathrm{C}=\mathrm{O}$ of thiouracil), 1,148, 1,337 $\left(\mathrm{SO}_{2}\right)$, 1,271 (C=S); ${ }^{1} \mathrm{H}$ NMR (DMSO- $\left.d_{6}\right), 2.3,2.5$ (2 H3C-S), 6.8 (1H, s, thiouracil), 7.2-7.4 (4H, dd), 9.8, $10.8\left(2 \mathrm{NH}\right.$, s, exchangeable with $\left.\mathrm{D}_{2} \mathrm{O}\right)$; MS: $m / z 312.4\left(\mathrm{M}^{+}\right.$, $33.05 \%)$.

\section{$4 a$}

Yield $80 \%$, m.p. $276{ }^{\circ} \mathrm{C}$, Anal. Calcd. $\mathrm{C}_{11} \mathrm{H}_{10} \mathrm{~N}_{3} \mathrm{O}_{3} \mathrm{~S}_{2} \mathrm{Br}$ (376.27): C, 35.11; $\mathrm{H}$, 2.68; N, 11.17. Found: C, $35.07 \mathrm{H}, 2.57$; N, 11.38; IR $\left(\mathrm{KBr}, \mathrm{cm}^{-1}\right): 3,355(\mathrm{NH})$, 2,968 ( $\mathrm{CH}$ aliphatic), 3,168 ( $\mathrm{CH}$ aromatic), 1,678 ( $\mathrm{C}=\mathrm{O}$ of thiouracil), 1,139, 1,320 $\left(\mathrm{SO}_{2}\right), 1,274(\mathrm{C}=\mathrm{S}) ;{ }^{1} \mathrm{H}$ NMR (DMSO- $\left.d_{6}\right), 2.4\left(\mathrm{H}_{3} \mathrm{C}-\mathrm{S}\right), 7.1-7.3(5 \mathrm{H}, \mathrm{m}), 9.9,10.4$ $\left(2 \mathrm{NH}, \mathrm{s}\right.$, exchangeable with $\left.\mathrm{D}_{2} \mathrm{O}\right)$; MS: $m / z 376.3\left(\mathrm{M}^{+}, 18.1 \%\right), 378.2(\mathrm{M}+2)^{+}$ $(17.4 \%)$.

\section{$4 b$}

Yield $69 \%$, m.p. $298{ }^{\circ} \mathrm{C}$, Anal. Calcd. $\mathrm{C}_{12} \mathrm{H}_{12} \mathrm{~N}_{3} \mathrm{O}_{3} \mathrm{~S}_{2} \mathrm{Br}$ (390.28): C, 36.92; H, 3.10; N, 10.77. Found: C, 37.01; H, 3.21; N, 10.82; IR (KBr, $\left.\mathrm{cm}^{-1}\right): 3,350(\mathrm{NH})$, 2,968 (CH aliphatic), 3,139 ( $\mathrm{CH}$ aromatic), 1,685 (C=O of thiouracil), 1,142, 1,328 $\left(\mathrm{SO}_{2}\right) ;{ }^{1} \mathrm{H}$ NMR (DMSO- $\left.d_{6}\right), 2.3,2.4\left(2 \mathrm{H}_{3} \mathrm{C}-\mathrm{S}\right), 7.2,7.3(4 \mathrm{H}, \mathrm{dd}), 9.8,10.2(2 \mathrm{NH}$, s, exchangeable with $\left.\mathrm{D}_{2} \mathrm{O}\right)$; MS: $m / z 390.3\left(\mathrm{M}^{+}, 26.2 \%\right), 392.7(\mathrm{M}+2)^{+}(25.7 \%)$.

\section{$5 a$}

Yield $70 \%$, m.p. $253{ }^{\circ} \mathrm{C}$, Anal. Calcd. $\mathrm{C}_{12} \mathrm{H}_{9} \mathrm{~N}_{3} \mathrm{O}_{4} \mathrm{~S}_{2}$ (323.34): C, 44.57; H, 2.81; N, 12.99. Found: C, 44.38; H, 2.97; N, 13.07; IR (KBr, $\left.\mathrm{cm}^{-1}\right): 3,300(\mathrm{NH}), 3,154$ ( $\mathrm{CH}$ aromatic), 1,668 ( $\mathrm{C}=\mathrm{O}$ of pyrimidine), 1,723 ( $\mathrm{C}=\mathrm{O}$ of thiazole), 1,140, 1,320 $\left(\mathrm{SO}_{2}\right) ;{ }^{1} \mathrm{H}$ NMR (DMSO- $\left.d_{6}\right), 2.2$ (2H of thiazole) $6.8(1 \mathrm{H}, \mathrm{s}$, pyrimidine), 7.1-7.4 $(5 \mathrm{H}, \mathrm{m}), 9.8\left(1 \mathrm{H}, \mathrm{s}\right.$, exchangeable with $\left.\mathrm{D}_{2} \mathrm{O}\right)$; $\mathrm{MS}: \mathrm{m} / \mathrm{z} 323.3\left(\mathrm{M}^{+}, 16.1 \%\right)$.

\section{$5 b$}

Yield $68 \%$, m.p. $280{ }^{\circ} \mathrm{C}$, Anal. Calcd. $\mathrm{C}_{13} \mathrm{H}_{11} \mathrm{~N}_{3} \mathrm{O}_{4} \mathrm{~S}_{2}$ (337.38): C, 46.28; H, 3.29; N, 12.45. Found: C, 46.19; H, 3.40; N, 12.62; IR (KBr, cm $\left.{ }^{-1}\right): 3,320(\mathrm{NH}), 3,164(\mathrm{CH}$ aromatic), 1,688 ( $\mathrm{C}=\mathrm{O}$ of pyrimidine), 1,721 ( $\mathrm{C}=\mathrm{O}$ of thiazole $), 1,140,1,320\left(\mathrm{SO}_{2}\right)$; ${ }^{1} \mathrm{H}$ NMR (DMSO- $d_{6}$ ), 2.2 (2H of thiazole), 2.5 (3H, s), 6.8 (1H, s, pyrimidine), 7.1-7.3 $(4 \mathrm{H}, \mathrm{dd}), 9.7\left(1 \mathrm{H}, \mathrm{s}\right.$, exchangeable with $\left.\mathrm{D}_{2} \mathrm{O}\right) ; \mathrm{MS}: \mathrm{m} / z 337.4\left(\mathrm{M}^{+}, 12.56 \%\right)$.

\section{$6 a$}

Yield $59 \%$, m.p. $308{ }^{\circ} \mathrm{C}$, Anal. Calcd. $\mathrm{C}_{20} \mathrm{H}_{15} \mathrm{~N}_{3} \mathrm{O}_{4} \mathrm{~S}_{2}$ (425.48): C, 56.45; H, 3.55; N, 9.88. Found: C, 56.67; H, 3.47; N, 9.97; IR (KBr, cm $\left.{ }^{-1}\right): 3,354(\mathrm{NH}), 3,109(\mathrm{CH}$ 
aromatic), 1,680 $(\mathrm{C}=\mathrm{O}$ of pyrimidine $), 1,728(\mathrm{C}=\mathrm{O}$ of thiazole $), 1,140,1,320\left(\mathrm{SO}_{2}\right)$; ${ }^{1} \mathrm{H}$ NMR (DMSO-d 6 ), 2.1 (3H, s, CH3), $6.2(1 \mathrm{H}, \mathrm{s}, \mathrm{C}=\mathrm{CH}), 6.7$ (1H, s, pyrimidine), 7.1-7.7 $(9 \mathrm{H}, \mathrm{m}), 9.5\left(1 \mathrm{H}, \mathrm{s}\right.$, exchangeable with $\left.\mathrm{D}_{2} \mathrm{O}\right) ; \mathrm{MS}: \mathrm{m} / \mathrm{z}(\%) 425.5(\mathrm{M})^{+}$ $(70.53 \%)$.

\section{6b}

Yield $73 \%$, m.p. $319{ }^{\circ} \mathrm{C}$, Anal. Calcd. $\mathrm{C}_{21} \mathrm{H}_{17} \mathrm{~N}_{3} \mathrm{O}_{4} \mathrm{~S}_{2}$ (439.51): C, 57.34; H, 3.89; N, 9.56. Found: C, 57.44; H, 3.87; N, 9.48; IR $\left(\mathrm{KBr}, \mathrm{cm}^{-1}\right): 3,377(\mathrm{NH}), 3,137(\mathrm{CH}$ aromatic), 1,685 ( $\mathrm{C}=\mathrm{O}$ of pyrimidine $), 1,730(\mathrm{C}=\mathrm{O}$ of thiazole $), 1,140,1,320\left(\mathrm{SO}_{2}\right)$; ${ }^{1} \mathrm{H}$ NMR (DMSO-d $\left.d_{6}\right), 2.1,2.2\left(6 \mathrm{H}, \mathrm{s}, 2 \mathrm{CH}_{3}\right), 6.2(1 \mathrm{H}, \mathrm{s}, \mathrm{C}=\mathrm{CH}), 6.9(1 \mathrm{H}, \mathrm{s}$, pyrimidine), 7.4-7.7 $(8 \mathrm{H}, \mathrm{m}), 9.4\left(1 \mathrm{H}, \mathrm{s}\right.$, exchangeable with $\left.\mathrm{D}_{2} \mathrm{O}\right) ; \mathrm{MS}: \mathrm{m} / z 439.5$ $\left(\mathrm{M}^{+}, 48.09 \%\right)$.

\section{$7 a$}

Yield $77 \%$, m.p. $325{ }^{\circ} \mathrm{C}$, Anal. Calcd. $\mathrm{C}_{12} \mathrm{H}_{9} \mathrm{~N}_{3} \mathrm{O}_{4} \mathrm{~S}_{2}$ (323.34): C, 44.57; H, 2.81; N, 12.99. Found: C, 44.56; H, 2.67; N, 13.20; IR (KBr, $\left.\mathrm{cm}^{-1}\right): 3,320(\mathrm{NH}), 3,174$ ( $\mathrm{CH}$ aromatic), 1,670 ( $\mathrm{C}=\mathrm{O}$ of pyrimidine), 1,727 $(\mathrm{C}=\mathrm{O}$ of thiazole $), 1,145,1,323$ $\left(\mathrm{SO}_{2}\right) ;{ }^{1} \mathrm{H}$ NMR (DMSO-d 6 ) 2.4 (2H of thiazole), 6.9 (1H, s, pyrimidine), 7.1-7.3 $(5 \mathrm{H}, \mathrm{m}), 9.8\left(1 \mathrm{H}, \mathrm{s}\right.$, exchangeable with $\left.\mathrm{D}_{2} \mathrm{O}\right)$; MS: $m / z(\%) 323.3(\mathrm{M})^{+}(10.76 \%)$.

\section{$7 b$}

Yield $65 \%$, m.p. $372{ }^{\circ} \mathrm{C}$, Anal. Calcd. $\mathrm{C}_{13} \mathrm{H}_{11} \mathrm{~N}_{3} \mathrm{O}_{4} \mathrm{~S}_{2}$ (337.38): C, 46.28; H, 3.29; N, 12.45. Found: C, 46.37; H, 3.40; N, 12.58; IR $\left(\mathrm{KBr}, \mathrm{cm}^{-1}\right): 3,300(\mathrm{NH}), 3,157$ ( $\mathrm{CH}$ aromatic), 1,685 ( $\mathrm{C}=\mathrm{O}$ of pyrimidine), 1,722 $(\mathrm{C}=\mathrm{O}$ of thiazole $), 1,140,1,320$ $\left(\mathrm{SO}_{2}\right) ;{ }^{1} \mathrm{H}$ NMR (DMSO-d $), 2.3\left(2 \mathrm{H}\right.$ of thiazole), $2.5\left(3 \mathrm{H}, \mathrm{s}, \mathrm{CH}_{3}\right), 6.8(1 \mathrm{H}, \mathrm{s}$, pyrimidine), 7.1-7.4 (4H, dd), $9.7\left(1 \mathrm{H}, \mathrm{s}\right.$, exchangeable with $\left.\mathrm{D}_{2} \mathrm{O}\right)$; $\mathrm{MS}: \mathrm{m} / z 337.4$ $\left(\mathrm{M}^{+}, 33.09 \%\right)$ (Scheme 1).

$\mathrm{N}$-(2-Hydrazinyl-6-oxo-1,6-dihydropyrimidin-4-yl)benzenesulfonamide and $\mathrm{N}$ (2-hydrazinyl-6-oxo-1,6-dihydropyrimidin-4-yl)-4-methylbenzenesulfonamide $(\mathbf{8 a}, \mathbf{b})$

A mixture of $\mathbf{2 a}, \mathbf{b}(0.005 \mathrm{~mol})$ and hydrazine hydrate $99 \%(0.005 \mathrm{~mol})$ in $30 \mathrm{ml}$ methanol was refluxed for $30 \mathrm{~h}$, then cooled and poured into ice/water; the produced solid was filtered off, dried, and crystallized from methanol.

$N$-(5-Oxo-1,5-dihydro[1,2,4]triazolo[4,3-a]pyrimidin-7-yl)benzenesulfonamide and 4-methyl- $N$-(5-oxo-1,5-dihydro[1,2,4]triazolo[4,3-a]pyrimidin-7-

yl)benzenesulfonamide $(\mathbf{9 a}, \mathbf{b})$

A mixture of $8 \mathbf{a}, \mathbf{b}(0.001 \mathrm{~mol})$ and $30 \mathrm{ml}$ formic acid was refluxed for $8 \mathrm{~h}$, then cooled and poured into ice/water; the precipitate was filtered off, dried, and crystallized from methanol. 


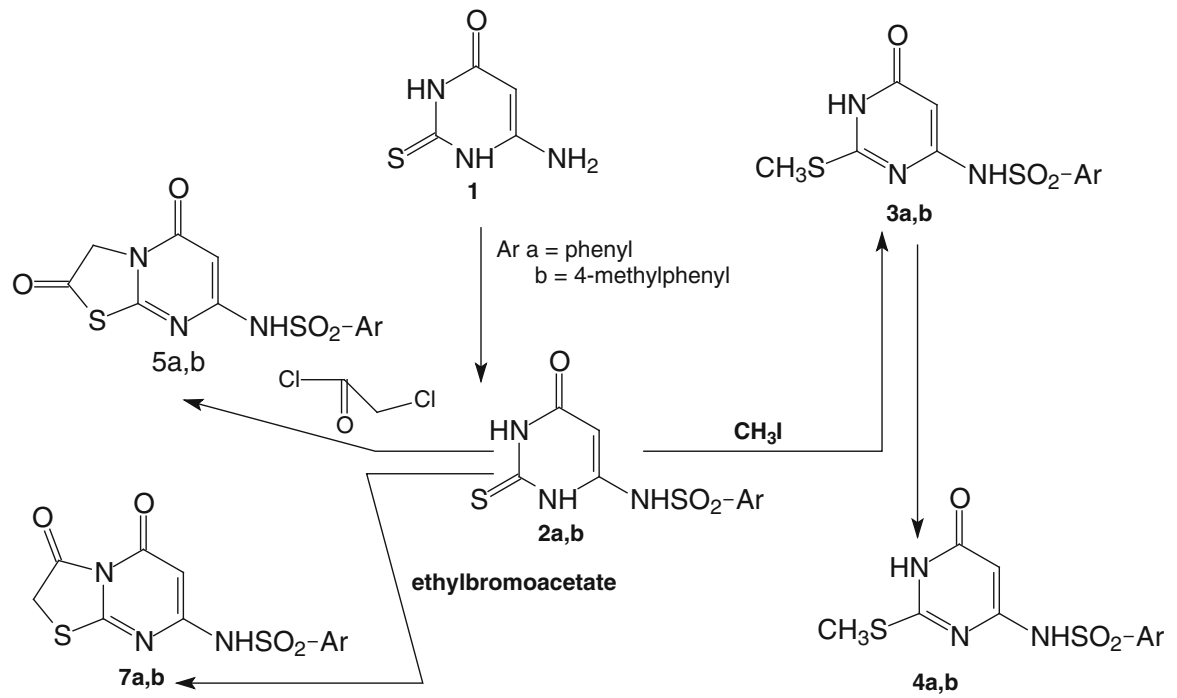

Scheme 1 The structure of compounds: $\mathbf{3 a}, \mathbf{b}-\mathbf{7 a}, \mathbf{b}$

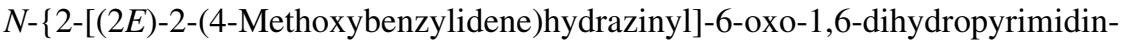
4-yl $\}-4-m e t h y l b e n z e n e s u l f o n a m i d e ~ a n d ~ N-\{2-[(2 E)-2-(4-$ methoxybenzylidene)hydrazinyl]-6-oxo-1,6-dihydropyrimidin-4yl benzenesulfonamide $(\mathbf{1 0 a}, \mathbf{b})$

A mixture of $\mathbf{8 a}, \mathbf{b}(0.001 \mathrm{~mol})$ and $p$-anisaldehyde $(0.001 \mathrm{~mol})$ in $30 \mathrm{ml}$ methanol was refluxed for $8 \mathrm{~h}$, then cooled, and the produced precipitate was filtered off, dried, and crystallized from DMF/water.

[(4-\{[Phenylsulfonyl]amino $\}-6-o x o-1,6-d i h y d r o p y r i m i d i n-2-y l) s u l f a n y l]$ acetic acid and [(4-\{[(4-methylphenyl)sulfonyl]amino $\}$-6-oxo-1,6-dihydropyrimidin-2yl)sulfanyl]acetic acid (11a, b)

To a solution of monochloroacetic acid $(0.03 \mathrm{~mol})$ and sodium hydroxide $(0.03 \mathrm{~mol})$ in $5 \mathrm{ml}$ water, a solution of $\mathbf{2 a}, \mathbf{b}(0.03 \mathrm{~mol})$ and sodium hydroxide $(0.03 \mathrm{~mol})$ was added. The reaction mixture was stirred at ambient temperature for $3 \mathrm{~h}$, then rendered acidic with hydrochloric acid $(6 \mathrm{~N})$. The separated solid was filtered off, washed with water, dried, and crystallized from methanol.

\section{$8 a$}

Yield $61 \%$, m.p. $277{ }^{\circ} \mathrm{C}$, Anal. Calcd. $\mathrm{C}_{10} \mathrm{H}_{11} \mathrm{~N}_{5} \mathrm{O}_{3} \mathrm{~S}$ (281.13): C, 42.72; H, 3.94; N, 24.91. Found: C, $42.75 \mathrm{H}, 3.70$; N, 24.83; IR (KBr, cm $\left.{ }^{-1}\right): 3,301(\mathrm{NH}), 3,167$ (CH aromatic), 1,665 ( $\mathrm{C}=\mathrm{O}$ of pyrimidine), 1,143, 1,325 $\left(\mathrm{SO}_{2}\right) ;{ }^{1} \mathrm{H}$ NMR (DMSO$\left.d_{6}\right), 3.1,6.2,6.8,10.5\left(\mathrm{NH}_{2}, 3 \mathrm{NH}, \mathrm{s}\right.$, exchangeable with $\left.\mathrm{D}_{2} \mathrm{O}\right), 6.9(1 \mathrm{H}, \mathrm{s}$, pyrimidine), 7.1-7.3 (5H, m); MS: $m / z 281.1\left(\mathrm{M}^{+}, 18.95 \%\right)$. 
$8 b$

Yield $70 \%$, m.p. $253{ }^{\circ} \mathrm{C}$, Anal. Calcd. $\mathrm{C}_{11} \mathrm{H}_{13} \mathrm{~N}_{5} \mathrm{O}_{3} \mathrm{~S}$ (295.32): C, 44.73; H, 4.44; N, 23.72. Found: C, 44.58; H, 4.37; N, 23.86; IR $\left(\mathrm{KBr}, \mathrm{cm}^{-1}\right): 3,312(\mathrm{NH}), 3,176$ (CH aromatic), 1,676 ( $\mathrm{C}=\mathrm{O}$ of pyrimidine), 1,143, 1,325 $\left(\mathrm{SO}_{2}\right) ;{ }^{1} \mathrm{H}$ NMR (DMSO$\left.d_{6}\right), 3.2,6.4,6.8,10.3\left(\mathrm{NH}_{2}, 3 \mathrm{NH}, \mathrm{s}\right.$, exchangeable with $\left.\mathrm{D}_{2} \mathrm{O}\right), 2.4\left(3 \mathrm{H}, \mathrm{s}, \mathrm{CH}_{3}\right), 6.7$ (1H, s, pyrimidine), 7.1-7.4 (4H, dd); MS: $m / z 295.3\left(\mathrm{M}^{+}, 54.94 \%\right)$.

\section{$9 a$}

Yield $66 \%$, m.p. $267{ }^{\circ} \mathrm{C}$, Anal. Calcd. $\mathrm{C}_{11} \mathrm{H}_{9} \mathrm{~N}_{5} \mathrm{O}_{3} \mathrm{~S}$ (291.30): C, 45.35; H, 3.11; N, 24.05. Found: C, 45.07; H, 3.45; N, 23.97; IR $\left(\mathrm{KBr}, \mathrm{cm}^{-1}\right): 3,354(\mathrm{NH}), 2,978$, 3,168 ( $\mathrm{CH}$ aromatic), 1,675 ( $\mathrm{C}=\mathrm{O}$ of pyrimidine), 1,140, 1,327 $\left(\mathrm{SO}_{2}\right)$; ${ }^{1} \mathrm{H}$ NMR (DMSO- $\left.d_{6}\right), 6.9(1 \mathrm{H}, \mathrm{s}$, thiouracil), $7.1(1 \mathrm{H}, \mathrm{s}$, triazole $), 7.2-7.4(5 \mathrm{H}, \mathrm{m}), 8.1,9.5$ (2NH, s, exchangeable $\left.\mathrm{D}_{2} \mathrm{O}\right)$; MS: $m / z 291.3\left(\mathrm{M}^{+}, 25.98 \%\right)$.

\section{$9 b$}

Yield $59 \%$, m.p. $295{ }^{\circ} \mathrm{C}$, Anal. Calcd. $\mathrm{C}_{12} \mathrm{H}_{11} \mathrm{~N}_{5} \mathrm{O}_{3} \mathrm{~S}$ (305.32): C, 47.19; H, 3.63; N, 22.94. Found: C, 47.32; H, 3.57; N, 22.97; IR (KBr, $\left.\mathrm{cm}^{-1}\right): 3,300(\mathrm{NH}), 3,168$ ( $\mathrm{CH}$ aromatic), 1,679 ( $\mathrm{C}=\mathrm{O}$ of pyrimidine), 1,140, 1,327 $\left(\mathrm{SO}_{2}\right) ;{ }^{1} \mathrm{H}$ NMR (DMSO$\left.d_{6}\right), 2.5\left(3 \mathrm{H}, \mathrm{s}, \mathrm{CH}_{3}\right), 6.9$ (1H, s, pyrimidine), $7.0(1 \mathrm{H}, \mathrm{s}$, triazole), 7.1-7.3 (4H, dd), 8.2, $9.4\left(2 \mathrm{NH}\right.$, s, exchangeable with $\left.\mathrm{D}_{2} \mathrm{O}\right)$; $\mathrm{MS}: \mathrm{m} / z 305.3\left(\mathrm{M}^{+}, 9.85 \%\right)$.

\section{$10 a$}

Yield $74 \%$, m.p. $321{ }^{\circ} \mathrm{C}$, Anal. Calcd. $\mathrm{C}_{18} \mathrm{H}_{17} \mathrm{~N}_{5} \mathrm{O}_{4} \mathrm{~S}$ (399.43): C, 54.12; H, 4.29; N, 17.53. Found: C, 54.07; H, 4.35; N, 17.25; IR $\left(\mathrm{KBr}, \mathrm{cm}^{-1}\right): 3,391(\mathrm{NH}), 3,197$ ( $\mathrm{CH}$ aromatic), 1,675 (C=O of pyrimidine), 1,145, 1,328 $\left(\mathrm{SO}_{2}\right) ;{ }^{1} \mathrm{H}$ NMR (DMSO$\left.d_{6}\right), 4.1\left(3 \mathrm{H}, \mathrm{s}, \mathrm{OCH}_{3}\right), 6.1(1 \mathrm{H}, \mathrm{s}, \mathrm{CH}=\mathrm{N}-), 6.2,6.8,10.5(3 \mathrm{NH}, \mathrm{s}$, exchangeable with $\left.\mathrm{D}_{2} \mathrm{O}\right), 6.9\left(1 \mathrm{H}, \mathrm{s}\right.$, pyrimidine), 7.1-7.4 (9H, m); MS: m/z $399.4\left(\mathrm{M}^{+}, 7.93 \%\right)$.

\section{$10 b$}

Yield $67 \%$, m.p. $347{ }^{\circ} \mathrm{C}$, Anal. Calcd. $\mathrm{C}_{19} \mathrm{H}_{19} \mathrm{~N}_{5} \mathrm{O}_{4} \mathrm{~S}$ (413.45): C, 55.19; H, 4.63; N, 16.94. Found: C, 55.50; H, 4.57; N, 16.76; IR (KBr, $\left.\mathrm{cm}^{-1}\right): 3,388(\mathrm{NH}), 3,165$ (CH aromatic), 1,687 ( $\mathrm{C}=\mathrm{O}$ of pyrimidine), 1,140, 1,326 $\left(\mathrm{SO}_{2}\right) ;{ }^{1} \mathrm{H}$ NMR (DMSO$\left.d_{6}\right), 2.5\left(3 \mathrm{H}, \mathrm{s}, \mathrm{CH}_{3}\right) 4.2\left(3 \mathrm{H}, \mathrm{s}, \mathrm{OCH}_{3}\right), 6.2(1 \mathrm{H}, \mathrm{s}, \mathrm{CH}=\mathrm{N}-), 6.1,6.8,10.4(3 \mathrm{NH}, \mathrm{s}$, exchangeable with $\left.\mathrm{D}_{2} \mathrm{O}\right), 6.9(1 \mathrm{H}, \mathrm{s}$, pyrimidine $), 7.1-7.3(8 \mathrm{H}, \mathrm{m}) ; \mathrm{MS}: \mathrm{m} / z 413.5$ $\left(\mathrm{M}^{+}, 12.07 \%\right)$.

\section{$11 a$}

Yield $68 \%$, m.p. $335{ }^{\circ} \mathrm{C}$, Anal. Calcd. $\mathrm{C}_{12} \mathrm{H}_{11} \mathrm{~N}_{3} \mathrm{O}_{5} \mathrm{~S}_{2}$ (341.36): C, 42.22; H, 3.25; $\mathrm{N}, 12.31$. Found: C, 42.58; H, 2.98; N, 12.45; IR $\left(\mathrm{KBr}, \mathrm{cm}^{-1}\right): 3,405(\mathrm{OH})$ very broad due to hydrogen bonding, 3,300 (NH), 2,958 (CH- aliphatic), 3,167 (CH 
aromatic), 1,675 (C=O of thiouracil), 1,750 (CO of $\mathrm{COOH}), 1,143,1,325\left(\mathrm{SO}_{2}\right) ;{ }^{1} \mathrm{H}$ NMR (DMSO-d $\left.d_{6}\right), 3.3\left(2 \mathrm{H}, \mathrm{s}, \mathrm{CH}_{2}\right), 6.9(1 \mathrm{H}, \mathrm{s}$, pyrimidine), 7.1-7.4 (5H, m), 9.9, $10.5\left(2 \mathrm{NH}, \mathrm{s}\right.$, exchangeable with $\left.\mathrm{D}_{2} \mathrm{O}\right), 11.7(1 \mathrm{H}, \mathrm{s}, \mathrm{COOH}) ; \mathrm{MS}: \mathrm{m} / z 341.4\left(\mathrm{M}^{+}\right.$, $19.87 \%)$.

\section{$11 b$}

Yield $60 \%$, m.p. $359{ }^{\circ} \mathrm{C}$, Anal. Calcd. $\mathrm{C}_{13} \mathrm{H}_{13} \mathrm{~N}_{3} \mathrm{O}_{5} \mathrm{~S}_{2}$ (355.39): C, 43.93; H, 3.69; $\mathrm{N}, 11.82$. Found: C, 43.69; H, 3.78; N, 11.78; IR $\left(\mathrm{KBr}, \mathrm{cm}^{-1}\right): 3,400(\mathrm{OH})$ very broad due to hydrogen bonding, 3,300 (NH), 2,960 (CH- aliphatic), 3,100 (CH aromatic), 1,670 $\left(\mathrm{C}=\mathrm{O}\right.$ of thiouracil), 1,754 $(\mathrm{C}=\mathrm{O}$ of $\mathrm{COOH}), 1,140,1,322\left(\mathrm{SO}_{2}\right)$; ${ }^{1} \mathrm{H}$ NMR (DMSO- $\left.d_{6}\right), 2.5\left(3 \mathrm{H}, \mathrm{s}, \mathrm{CH}_{3}\right), 3.4\left(2 \mathrm{H}, \mathrm{s}, \mathrm{CH}_{2}\right), 6.7$ (1H, s, pyrimidine), 7.2-7.4 (4H, dd), 9.8, $10.3\left(2 \mathrm{NH}, \mathrm{s}\right.$, exchangeable with $\left.\mathrm{D}_{2} \mathrm{O}\right), 11.8(1 \mathrm{H}, \mathrm{s}, \mathrm{COOH})$; MS: $m / z 355.4\left(\mathrm{M}^{+}, 23.84 \%\right)$ (Scheme 2).

\section{Biological investigation}

On the basis of monitoring the inhibition of the growth of human cancer cells, a series of novel pyrimidine derivatives possessing a broader spectrum of antitumor activity and fewer toxic side-effects than traditional anticancer drugs have been studied.

Ten selected pyrimidine derivatives (3a, 3b, 4a, 5b, 6a, 7b, 8a, 9b, 10a, and 11a) were subjected to screening for investigation of their antiproliferative potency against human A549 (lung), MCF-7 (breast), HT-29 (colon), and HEPG2 (liver) cancer cell lines.

The results indicate that the cell lines most sensitive to the antiproliferative activity of tested compounds are human liver HEPG2 and colon HT-29. In the case of human liver HEPG2, compounds $4 \mathbf{a}, 3 \mathbf{a}, \mathbf{5 a}$, and 10a showed the highest

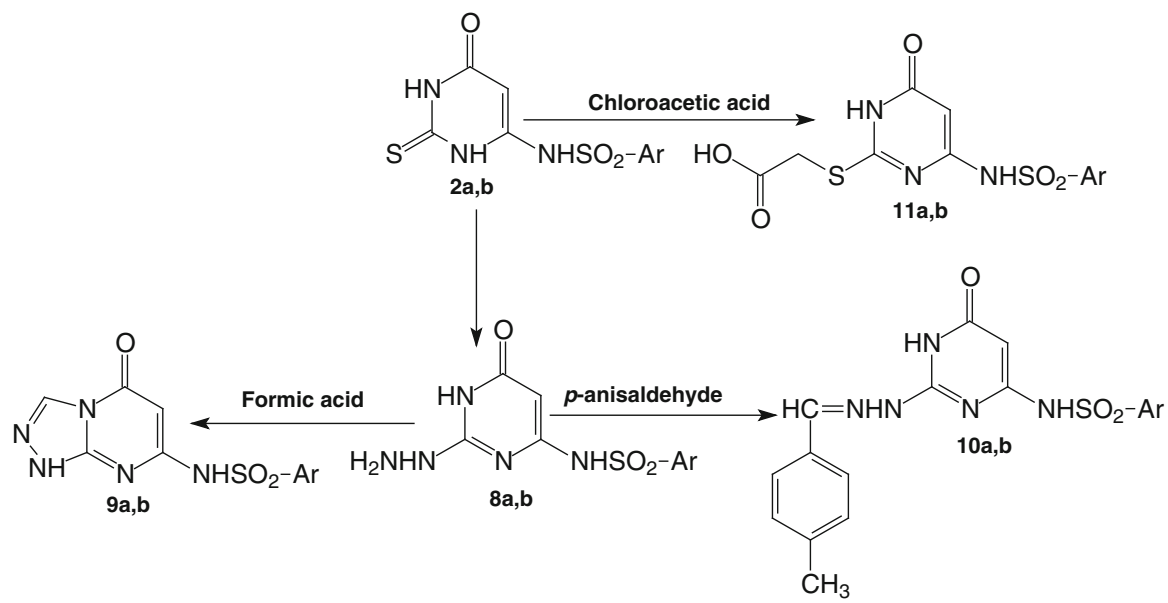

Scheme 2 The structure of compounds: 8a,b-11a,b 
antiproliferative activity compared with other compounds. On the other hand, in the case of colon cancer HT-29, only compound 4a showed moderate activity in comparison with other compounds.

In this study, we have identified some pyrimidine derivatives as a novel class of compounds with antiproliferative activity. The lead compounds were the most potent in the biological assay employed (showing, e.g., improved growth inhibition potential as compared with the reference anticancer drugs).

These experimental findings may provide support for use of these novel compounds as new weapons in the fight against different types of cancer.

\section{Materials and methods}

Cells

\section{Cell lines}

Two groups of cell lines were used in this work: (1) The first group: A549 (human lung cancer), MCF-7 (human breast cancer), and HT-29 (human colon cancer) are maintained in the Institute of Immunology and Experimental Therapy, Wroclaw, Poland. Cell lines A549 and HT-29 were cultured in medium Opti-MEM (Gibco, Scotland, USA) and RPMI 1640 (IIET, Wroclaw, Poland) supplemented with $2 \mathrm{mM} \mathrm{L-}$ glutamine, fetal bovine serum (5\%) (all from Sigma-Aldrich Chemie $\mathrm{GmbH}$, Steinheim, Germany), $100 \mathrm{U} / \mathrm{ml}$ penicillin and $100 \mu \mathrm{g} / \mathrm{ml}$ streptomycin (both from Polfa Tarchomin S.A., Warsaw, Poland), and $1 \mathrm{mM}$ sodium pyruvate (from SigmaAldrich Chemie GmbH, Steinheim, Germany), only in case of HT-29 cell line. Cell line MCF-7 was cultured in Eagle medium (IIET, Wroclaw, Poland) supplemented with $2 \mathrm{mM}$ L-glutamine, $0.01 \mathrm{mg} / \mathrm{ml}$ insulin, $1 \mathrm{mM}$ sodium pyruvate, fetal bovine serum (10\%) (all from Sigma-Aldrich Chemie GmbH, Steinheim, Germany), and $100 \mathrm{U} / \mathrm{ml}$ penicillin and $100 \mu \mathrm{g} / \mathrm{ml}$ streptomycin (both from Polfa Tarchomin S.A., Warsaw, Poland). All lines were obtained from the American Type Culture Collection (Rockville, MD, USA). Cells were cultured at $37{ }^{\circ} \mathrm{C}$ in humid atmosphere saturated with $5 \% \mathrm{CO}_{2}$.

(2) The second group: HEPG2 (human liver cancer cell line), is maintained at the National Institute of Cancer, Cairo University, Cairo, Egypt.

\section{Compounds}

Prior to usage, the newly synthesized compounds $(3 \mathbf{3 a}, \mathbf{3 b}, \mathbf{4 a}, \mathbf{5 b}, \mathbf{6 a}, \mathbf{7 b}, \mathbf{8 a}, \mathbf{9 b}$, 10a, and 11a) were dissolved in DMSO and culture medium (1:9) to concentration of $1 \mathrm{mg} / \mathrm{ml}$ and subsequently diluted in culture medium to reach the required concentrations (ranging from 1 to $100 \mu \mathrm{g} / \mathrm{ml}$ ).

Antiproliferative assay in vitro

At $24 \mathrm{~h}$ before addition of the tested compounds, the cells were placed in 96-well plates (Sarstedt, Germany) at density of $1 \times 10^{4}$ cells per well. An assay was 
performed after $72 \mathrm{~h}$ of exposure to varying concentrations of the tested agents. The in vitro cytotoxic effect of all agents was examined using the sulforhodamine B (SRB) assay [18].

\section{SRB cytotoxicity test}

Cells were attached to the bottom of plastic wells by fixing them with cold $50 \%$ trichloroacetic acid (TCA; Sigma-Aldrich Chemie GmbH, Steinheim, Germany) on the top of the culture medium in each well. The plates were incubated at $4{ }^{\circ} \mathrm{C}$ for $1 \mathrm{~h}$, then washed five times with tap water. The cellular material fixed with TCA was stained with $0.04 \%$ sulforhodamine B (SRB; Sigma-Aldrich Chemie GmbH, Steinheim, Germany) dissolved in $1 \%$ acetic acid (POCH, Gliwice, Poland) for $30 \mathrm{~min}$. Unbound dye was removed by rinsing $(4 \times)$ in $1 \%$ acetic acid. The proteinbound dye was extracted with $10 \mathrm{mM}$ unbuffered Tris base (POCH, Gliwice, Poland) for determination of the optical density $(\lambda=540 \mathrm{~nm})$ in a computerinterfaced, 96-well microtiter plate reader (Multiskan RC photometer; Labsystems, Helsinki, Finland).

The results were calculated as an $\mathrm{IC}_{50}$ (50\% inhibitory concentration) value, i.e., the concentration of tested agent which inhibits proliferation of $50 \%$ of the cancer cell population. IC values were calculated for each experiment separately, and mean values with standard deviation (SD) are presented in the table. Each compound at each concentration was tested in triplicate in a single experiment, which was repeated 3-7 times.

\section{Results}

Table 1 indicates the cytotoxic activity of the newly synthesized derivatives $\mathbf{3 a}, \mathbf{3} \mathbf{b}$, $\mathbf{4 a}, 5 \mathbf{b}, 6 \mathbf{a}, 7 \mathbf{b}, \mathbf{8 a}, 9 \mathbf{b}, \mathbf{1 0 a}$, and 11a against various cancer cell lines. Their activity was compared with the traditional anticancer drugs 5-FU and CIS. It can be deduced from the preliminary screening results that the cell lines most sensitive to the antiproliferative activity of tested compounds are human liver HEPG2 and colon cancer HT-29. All selected compounds exhibited moderate to strong growth inhibition activity against HEPG2 cell line with $\mathrm{IC}_{50}$ values ranging between 1 and $10 \mu \mathrm{g} / \mathrm{ml}$. The most active compounds, which revealed antiproliferative activity also against human colon HT-29 and breast MCF-7 cell lines, were 3a, 3b, 4a, and $10 a$.

\section{Discussion}

Cytotoxic drugs remain the mainstay of cancer chemotherapy, being administered in novel therapeutic approaches such as signal inhibition. It is therefore important to discover novel cytotoxic agents with spectra of activity and toxicity that differ from currently used agents. It is well known that chemotherapy aims to destroy cancer cells using various types of chemicals. The substances used are supposed to target 
Table 1 Antiproliferative activity of some selected newly synthesized compounds against different carcinoma cell lines

\begin{tabular}{|c|c|c|c|c|}
\hline \multirow[t]{2}{*}{ Compound } & \multicolumn{4}{|l|}{$\mathrm{IC}_{50}(\mu \mathrm{g} / \mathrm{ml})$} \\
\hline & A549 & MCF-7 & HT-29 & HEPG2 \\
\hline 3a & na & $51.69 \pm 8.0$ & $6.93 \pm 1.65$ & 4.1 \\
\hline 3b & $55.39 \pm 6.53$ & $40.51 \pm 8.26$ & $14.72 \pm 3.56$ & 3.92 \\
\hline $4 \mathbf{a}$ & na & $67.86 \pm 11.04$ & $46.99 \pm 9.21$ & 4.31 \\
\hline $5 \mathbf{a}$ & na & na & na & 9.38 \\
\hline $6 \mathbf{a}$ & na & na & na & 6.59 \\
\hline $7 b$ & na & na & na & 6.53 \\
\hline $\mathbf{8 a}$ & na & $54.41 \pm 8.70$ & na & 8.54 \\
\hline $9 b$ & na & na & na & 8.48 \\
\hline $10 \mathrm{a}$ & $41.77 \pm 9.13$ & $25.74 \pm 4.47$ & $14.35 \pm 3.54$ & 3.74 \\
\hline $11 \mathrm{a}$ & na & na & na & 7.43 \\
\hline Cisplatin & $4.08 \pm 0.43$ & $3.34 \pm 0.52$ & $4.30 \pm 3.67$ & 3.58 \\
\hline 5-Fluorouracil & $7.22 \pm 0.44$ & $3.65 \pm 0.93$ & $0.37 \pm 0.28$ & 5 \\
\hline DMSO & $\mathrm{na}^{*}$ & na* & $\mathrm{na}^{*}$ & na* \\
\hline
\end{tabular}

na not active at tested concentration (from 100 to $0.1 \mu \mathrm{g} / \mathrm{ml}$ ), $n a^{*}$ not active at the highest concentration of DMSO (1\% solution)

mainly cancer cells, and doses are calculated to minimize collateral damage to surrounding tissues, which nevertheless occurs. This type of treatment increases the entropy of the organism, suppresses the immune system, and forms a toxic cell environment which may destroy surrounding healthy cells. So, it is important to minimize effective doses to the least amount, as well as to try to minimize the sideeffects of these drugs. Therefore, novel pyrimidine derivatives with a comparable spectrum of antiproliferative activity to 5-FU, but potentially fewer side-effects, were developed. The antiproliferative activities of such compounds were assessed against the HEPG2 cancer cell line in comparison with the traditional anticancer drugs 5-FU and CIS. In the antiproliferative activity study, some of the selected compounds showed reasonable antiproliferative activity in comparison with 5-FU and CIS. Moreover, study of the biochemical parameters induced in mice by the tested compounds showed insignificant differences relative to the control group, which indicates a moderate margin of safety for the selected compounds. Compared with 5-FU and doxorubicin (DOX), dose augmentation of compounds 10a and 11a, in a search for possibly higher potency, therefore seems realizable without undesirable implications. Furthermore, the selected compounds have important potential advantages over 5-FU and DOX because of their lower toxicity and their ability to induce lower biochemical parameters. These results are in agreement with the work of many authors who have reported novel derivatives of 5-FU with a broader spectrum of antitumor activity and fewer toxic side-effects than 5-FU. 


\section{Conclusions}

Various compounds were selected for further structural modification in an effort to increase their potency. The goal of the present work is to explore the significance of the spacer for the respective biological anticancer activity. The anticancer activity data indicate that the cell lines most sensitive to the activity of tested compounds are human liver HEPG2 and colon cancer HT-29. In the case of human liver HEPG2, compounds 3a, 3b, 4a, and 10a showed the highest antiproliferative activity compared with other compounds. On the other hand, in the case of colon cancer HT29 , only compound 3a showed moderate activity in comparison with other compounds.

Acknowledgments The authors are grateful and offer their deep thanks to the members of the Therapeutic Chemistry Department, Pharmaceutical and Drug Industries Division, National Research Centre, and the Department of Experimental Oncology, Institute of Immunology and Experimental Therapy, 12, Rudolf Weigl St. 53-114, Wroclaw, Poland for helping them to do this work.

Open Access This article is distributed under the terms of the Creative Commons Attribution License which permits any use, distribution, and reproduction in any medium, provided the original author(s) and the source are credited.

\section{References}

1. C.A. Heidelbereger, K.C. Leibman, P.M. Bhargava, Cancer Res. 17, 39 (1957)

2. B.H. Longley, D.P. Harkin, P.G. Tohnston, Nat. Rev. Cancer 3(5), 330-338 (2003)

3. M.J. Straus, N.B. Mantel, A.H. Goldin, Cancer Res. 35, 126 (1962)

4. E.N. Sassenrath, A.M. Kells, D.M. Greenberg, Cancer Res. 19, 259 (1959)

5. M.A. Friedland, D.W. Visser, Biochem. Biophys. Acta 51, 148 (1961)

6. G.M. Vansanten, F.A. Sorm, E.H. Siman, J. Biol. Chem. 237, 1271 (1965)

7. R.K. Zahn, U.K. Hagen, Mol. Pharmac. 1, 113 (1972)

8. C.G. Tomisek, P.W. Todd, E.F. Borex, Canadian Cancer Res. Conference (Pergaman press, 1995), p. 34

9. T. Pospieszny, M. Szymankiewicz, E. Wyrzykiewicz, ISRN Org. Chem.2011, 6 (2011). Article ID 610521

10. S. Mosaad, S. Mohamed, M. Awad, S.I. Amira Ibrahim, Molecules 15, 1882-1890 (2010)

11. T.S. Chitre, K.G. Bothara, S.M. Patil, K.D. Asgaonkar, S. Nagappa, M.K. Kathiravan, Int. J. Res. Pharm. Biomed. Sci. 2(2), 616-623 (2011)

12. O.A. Fathalla, W.A. Zaghary, H.H. Radwan, S.M. Awad, M.S. Mohamed, Arch. Pharm. Res. 25(3), 258-269 (2002)

13. M. Nakamura, S. Jonsson, Arch. Biochem. Biophys. 66, 183-189 (1957)

14. V.J. Ram, Archiv der Pharmazie. 324(11), 837-839 (1991)

15. V.J. Ram, A.S. Goel, M.K. Nath, P.H. Srivastava, Prog. Drug Res. 52, 53-101 (1999)

16. C. Taylor, J. Edward, C. Cheng, J. Org. Chem. 25, 148 (1960)

17. S. Youssif, S. El-Bahaie, E. Nabih. Bull. Korean Chem. Soc. 24(10), 1429-1432 (2003)

18. C. Wiart, Evid. Based Complement Alternat. Med. 4(3), 299-311 (2007) 